\title{
A Construction of Dual Wavelet and its Applications in Image Edge Detection
}

\author{
Yubin Li \\ Gannan Normal University, Ganzhou, China \\ liyubin2201@163.com
}

\begin{abstract}
The edge detection is usually used for controlling the automation of pattern recognition in intelligent machines, traffic control and mobile devices security systems. Due to the complex of image control, the dimensions are increasing so that the single wavelet is not able to address some applications, thus, high-dimensional wavelet basis is needed. This paper introduces a construction of dual wavelet for fulfilling the gap. A dual wavelet through the compactly support set/functions which can form the Riesz basis is built up. Based on the mathematic presentation of the construction of the dual wavelet, which is a dual orthogonal with scale function and duality, the constructed dual wavelet is used for an application testing with fingerprint. The proposed approach is compared with Sobel and Canny filter. It is observed that from the results, the proposed dual wavelet outperforms the Sobel and Canny filter in terms of effectiveness and computation time.
\end{abstract}

Keywords: Wavelet, Dual, Orthogonal, Image Processing, Edge Detection

\section{Introduction}

Wavelet analysis is an emerging discipline which is rapidly developed over the last decade. It covers a broad impact of applications in real-life cases such as mathematical applied uses, quantum mechanics acoustic data processing, vision science, and other areas of computer science and technology, especially in signal processing, image processing, pattern recognition, and voice analysis [1]. In the wavelet theory, all wavelet transforms may be considered forms of time-frequency representation for continuous-time (analog) signals and so are related to harmonic analysis. Almost all practically useful discrete wavelet transforms use discrete-time filter banks [2]. These filter banks are called the wavelet and scaling coefficients in wavelets nomenclature. These filter banks may contain either finite impulse response (FIR) or infinite impulse response (IIR) filters [3]. The wavelets forming a continuous wavelet transform (CWT) are subject to the uncertainty principle of Fourier analysis respective sampling theory: Given a signal with some event in it, one cannot assign simultaneously an exact time and frequency response scale to that event.

The history of wavelet could be traced back to 1910 when Haar proposed the first orthogonal basis [4]. A significant breakthrough of the wavelet analysis was made by Meyer who created a smooth function with certain decay $\varphi$ [5]. The binary extension and movement $\left\{\varphi_{j}^{k}=2^{-j / 2} \varphi\left(2^{-j} \mathrm{t}-\mathrm{k}\right): \mathrm{j}, \mathrm{k} \in \square\right\}$ consist of the orthogonal basis of $L^{2}(\Re)$. The significance of this transform brings opportunities to realize wavelet in real applications. For example, in 1987, computer scientist Mallat with Meyer used the wavelet analysis in computer vision domain to analyze the multi-dimensional resolution in image processing [6].

As the complexity of real-life application is tremendously increasing, the wavelet analysis is developing rapidly. For example, the dimensions of the wavelet is increasing 
so as to meet the scientific applications from geographical cases, aerospace applications and so on. Due to the complexity of high dimensional wavelet basis, the construction of $n$ dimensional wavelet is very difficult. For the construction of non-tensor product of compactly supported wavelets, current studies are very limited [7]. In order to address the complex and huge data-based applications such as digital image processing, the compactly supported wavelets cannot performs well.

This paper introduces a construction of dual wavelet so as to address the highdimensional and non-constructed data applications such as digital virtual human image, biological image processing, and 3D objects recognition and tracing. Applications of the constructed dual wavelet are based on the image processing to presenting the effectiveness and efficiency by comparing with other approaches through the experiments of edge detection. The edge detection is usually used for controlling the automation of pattern recognition in intelligent machines, traffic control and mobile devices security systems [8]. Due to the complex of image control, the dimensions are increasing so that the single wavelet is not able to address some applications. High-dimensional wavelet basis is needed. The proposed dual wavelet is based on the advantages of biorthogonality which is used for constructing linear filter that could meet the high-dimensional applications [9-11].

The rest of this paper is organized as follows. Section 2 gives a basic concept of dual wavelet in terms of its construction and compactly supported set. Section 3 presents the construction of dual wavelet including the scaling function, validation, and duality of the dual wavelet. Section 4 demonstrates the numerical examples of the dual wavelets. Experiments and applications are discussed in section 5 through an image processing case. Specifically focus is placed on the edge detection. This section compares the proposed approach with other methods. Section 6 concludes this paper by giving the findings and future research directions.

\section{Dual Wavelet}

In the image processing with high dimensions, the precise decomposition and reconstruction is very important. The dual wavelet may be suitable for addressing this issue since the scale function could be orthogonal. The main idea of the dual wavelet is from the $S_{3 k+1}^{2 k}\left(\Delta_{m, n}^{1}\right)$ space where the dual orthogonal wavelet with non-tensor product supported set could be constructed. Firstly, Groebner Basis approach is used for getting the dual function $\phi$ of the scale function $\phi$. Secondly, using the restricted transition operator, the characteristics of Riesz and quadratic integrability of $\phi$ could be examined. Finally, under the scale and dual-scale functions, a matrix extension method is used for constructing the dual wavelet based on the scale and dual-scale functions.

Assume $\phi(x) \in L^{2}\left(\mathfrak{R}^{2}\right)$ is a compactly supported function, there is a limited sequence $\left\{\mathrm{h}_{j}\right\}_{j \in \square^{2}}$ s.t. $\phi(x)=\sum_{j \in \square^{2}} h_{j} \phi(2 x-j)$ then,

$$
\phi(w)=0.25 \sum_{j \in \square^{2}} h_{j} e^{-i w \llbracket j / 2} \phi(w / 2)
$$

Mark $M(z)=0.25 \sum_{j \in \square^{2}} h_{j} z^{j}$ is the two scale operator of $\phi$, where $z=e^{-\frac{i w}{2}}$.

Let $\phi(x)$ is the dual B basis in the space of $S_{3 k+1}^{2 k}\left(\Delta_{m, n}^{1}\right)$, an embedded space $V_{0} \subset V_{1} \ldots \subset V_{j} \subset V_{j+1} \ldots$ could be established by $\phi(x)$ in $L^{2}\left(\Re^{2}\right)$. Where,

$$
V_{j}=\overline{\operatorname{span}\left\{\phi\left(2^{j} x-l\right), l \in \square^{2}\right\}}, j \in \square
$$


The movement $\left\{\phi(\square-l), l \in \square^{2}\right\}$ of $\phi(x)$ forms the Riesz basis of $V_{0}$. $\exists 0<A \leq B<+\infty$, s.t.

$$
A\|a\|_{2} \leq\left\|\sum_{l \in \square^{2}} a_{l} \phi(x-l)\right\|_{2} \leq B\|a\|_{2}
$$

$\forall a=\left\{a_{l}\right\}_{l \in \square^{2}} \in l^{2}\left(\square^{2}\right), \quad \phi$ generates a multi-resolution analysis (MRA) of $L^{2}\left(\square^{2}\right)$. From $\phi$, it is possible to construct the dual orthogonal wavelet. The first step is to find out the compactly supported function $\phi$, which is dual orthogonal with $\phi$ :

$$
<\phi(x), \phi(x-l)>=\delta_{l, 0}, l \in \square^{2}
$$

For $\phi$, there is a limited sequence $\left\{h_{j}\right\}_{j \in \square^{2}}$, s.t.

$$
\begin{aligned}
& \phi(x)=\sum_{j \in \square^{2}} h_{j} \phi(2 x-j) \text {, then } \phi(w)=0.25 \sum_{j \in \coprod^{2}} h_{j} e^{\frac{-i j \llbracket w}{2}} \phi\left(\frac{w}{2}\right), \\
& \text { So } M(z)=0.25 \sum_{j \in \square^{2}} h_{j} z^{j}
\end{aligned}
$$

(5) is the dual scale symbol.

Lemma 1. Assume $\phi, \phi \in L^{2}\left(\square^{2}\right)$, then $\phi$ and $\phi$ is dual orthogonal.

Proof.

$$
\begin{aligned}
\delta_{l, 0} & =<\phi(x), \phi(x-l)> \\
& =\frac{1}{4 \pi^{2}}<\phi(w), \phi(w) e^{-i w \square}> \\
& =\frac{1}{4 \pi^{2}} \int_{\sqcup^{2}} \phi(w) \phi(w) e^{i w \square} d w \\
& =\frac{1}{4 \pi^{2}} \sum_{k \in \square^{2}} \int_{[0,2 \pi]^{2}} \phi(w+2 \pi k) \overline{\phi(w+2 \pi k)} e^{i w \square} d w \\
& =\frac{1}{4 \pi^{2}} \int_{[0,2 \pi]^{2}} e^{i w \square} \sum_{k \in \square^{2}} \phi \overline{(w+2 \pi k)} \overline{\phi(w+2 \pi k)} d w \\
& \Leftrightarrow \sum_{k \in \square^{2}} \phi(w+2 \pi k) \phi(w+2 \pi k)=1, \text { a.e. } \quad w \in \square^{2}
\end{aligned}
$$

Lemma 2. If $\sum_{k \in \square^{2}} \phi(w+2 \pi k) \phi(w+2 \pi k)=1 \quad$, a.e. $w \in \square^{2}, \quad$ then $\sum_{\varepsilon \in E} M\left((-1)^{\varepsilon} z\right) \overline{M\left((-1)^{\varepsilon} z\right)}=1$, where $\varepsilon=\left(\varepsilon_{1}, \varepsilon_{2}\right) \in E,\left((-1)^{\varepsilon} z\right)=\left((-1)^{\varepsilon_{1}} z_{1},(-1)^{\varepsilon_{2}} z_{2}\right)$.

Proof.

$$
\begin{aligned}
& 1=\sum_{k \in \prod^{2}} \phi(w+2 \pi k) \overline{\phi(w+2 \pi k)} \\
& =\sum_{k \in \square^{2}} M\left(e^{-i(w / 2+\pi k)}\right) \phi(w / 2+\pi k) \overline{M\left(e^{-i(w / 2+\pi k)}\right) \phi(w / 2+\pi k)} \\
& =\sum_{k \in \prod^{2}} M\left(e^{-i(w / 2+\pi k)}\right) \overline{M\left(e^{-i(w / 2+\pi k)}\right)} \phi(w / 2+\pi k) \overline{\phi(w / 2+\pi k)}
\end{aligned}
$$




$$
\begin{aligned}
=\sum_{\varepsilon \in E} \sum_{l \in \square^{2}} M\left(e^{-i(w / 2+\pi \varepsilon+2 \pi l)} \overline{M\left(e^{-i(w / 2+\pi \varepsilon+2 \pi l)}\right)} \phi(w / 2+\pi \varepsilon+2 \pi l) \overline{\phi(w / 2+\pi \varepsilon+2 \pi l)}\right. \\
=\sum_{\varepsilon \in E} \sum_{l \in \square^{2}} M\left(e^{-i(w / 2+\pi \varepsilon)} \overline{M\left(e^{-i(w / 2+\pi \varepsilon)}\right)} \phi(w / 2+\pi \varepsilon+2 \pi l) \overline{\phi(w / 2+\pi \varepsilon+2 \pi l)}\right. \\
=\sum_{\varepsilon \in E} M\left(e^{-i(w / 2+\pi \varepsilon)} \overline{M\left(e^{-i(w / 2+\pi \varepsilon)}\right)} \sum_{l \in \square^{2}} \phi(w / 2+\pi \varepsilon+2 \pi l) \overline{\phi(w / 2+\pi \varepsilon+2 \pi l)}\right. \\
=\sum_{\varepsilon \in E} M\left(e^{-i(w / 2+\pi \varepsilon)} \overline{M\left(e^{-i(w / 2+\pi \varepsilon)}\right)}\right. \\
=\sum_{\varepsilon \in E} M\left((-1)^{\varepsilon} z\right) \overline{M\left((-1)^{\varepsilon} z\right)}
\end{aligned}
$$

Construction of the dual orthogonal wavelet follows the steps: (1) find out the dual orthogonal function $\phi$ of $\phi .\left\{V_{j}\right\}_{j \in \square}, \phi$ and $\left\{V_{j}\right\}_{j \in \square}$ forms the MRA in $L^{2}\left(\square^{2}\right)$; (2) work out the $\varphi^{\varepsilon}$ in $V_{1}$ and $\varphi^{\varepsilon}$ in $V_{1}, \varepsilon \in E$, s.t.

$$
\begin{array}{ccc}
<\varphi^{\varepsilon}(x), \varphi^{\mu}(x-l)>=\delta_{l, 0} \delta_{\varepsilon, \mu} & \varepsilon, \mu \in E & l \in \square^{2} \\
<\phi(x), \varphi^{\varepsilon}(x-l)>=0 & \varepsilon \in E & l \in \square^{2} \\
<\varphi^{\varepsilon}(x), \phi^{\varepsilon}(x-l)>=0 & \varepsilon \in E & l \in \square^{2}
\end{array}
$$

The Riesz basis of $V_{1}$ is formed by $\left\{\phi(x-l), \varphi^{\varepsilon}(x-l), \varepsilon \in E, l \in \square^{2}\right\}$. The Riesz basis of $V_{1}$ is from $\left\{\phi(x-l), \varphi^{\varepsilon}(x-l), \varepsilon \in E, l \in \square^{2}\right\}$. Since $\varphi^{\varepsilon}(x) \in V_{1}, \varphi^{\varepsilon}(x) \in V_{1}$, then:

$$
\begin{gathered}
\varphi^{\varepsilon}(x)=\sum_{l \in \square^{2}} g_{l}^{\varepsilon} \phi(2 x-l), \varepsilon \in E \\
\varphi^{\varepsilon}(x)=\sum_{l \in \square^{2}} g_{l}^{\varepsilon} \phi(2 x-l), \varepsilon \in E
\end{gathered}
$$

We can get:

$$
\begin{gathered}
\varphi^{\varepsilon}(w)=M^{\varepsilon}(z) \phi(w / 2), \varepsilon \in E \\
\varphi^{\varepsilon}(w)=M^{\varepsilon}(z) \phi(w / 2), \varepsilon \in E
\end{gathered}
$$

Where,

$$
\begin{aligned}
& M^{\varepsilon}(z)=M^{\varepsilon}\left(z_{1}, z_{2}\right)=0.25 \sum_{l \in \square^{2}} g_{l}^{\varepsilon} z^{l}, \varepsilon \in E \\
& M^{\varepsilon}(z)=M^{\varepsilon}\left(z_{1}, z_{2}\right)=0.25 \sum_{l \in \square^{2}} g_{l}^{\varepsilon} z^{l}, \varepsilon \in E
\end{aligned}
$$

\section{Construction of Dual Wavelet}

The construction of dual wavelet is firstly to work out the dual scale function $\varphi$, which could be obtained when the $M(z)$ meets the condition $\sum_{\varepsilon \in E} M\left((-1)^{\varepsilon} z\right) \overline{M\left((-1)^{\varepsilon} z\right)}=1$. Then, the dual wavelet is converted $M(z)$ into $q_{\varepsilon}, \varepsilon \in E$, s.t. $\sum_{\varepsilon \in E} p_{\varepsilon}\left(z^{2}\right) q_{\varepsilon}\left(z^{2}\right)=0.25$. The following sections reports on the 
establishing of the scaling function and validation of $\mathrm{H}\left(\mathrm{z}_{1}, \mathrm{z}_{2}\right)$. The duality of the constructed wavelet is presented as well in this section.

\subsection{Scaling Function}

For considering a binary polynomial $f_{1}, f_{2}, \ldots, f_{s} \in K[z]$, if there is a binary polynomial $g_{1}, g_{2}, \ldots, g_{s} \in K[z]$, s.t. $\sum_{i=1}^{s} f_{i} g_{i}=1$, where $z \in \square^{2}, K[z]$ is the polynomial circle in complex domain. Based on the Buchberger algorithm for getting Groebner basis, the Hilbert Nullstellensatz theorem can get, given an $I$, $I=K[z] \Leftrightarrow\{1\}$, which is the Greobner basis of $I$.

Lemma 3. If $f_{1}, f_{2}, \ldots, f_{s} \in K[z]$ has no public Zero point in $\square^{2}$, $g_{1}, g_{2}, \ldots, g_{s} \in K[z]$ exists, s.t. $\sum_{i=1}^{s} f_{i} g_{i}=1$.

Proof. Assume that $f_{i}, i=1,2, \ldots s$ is sequenced by $z_{1}>z_{2}$. Let $G=\left\{h_{1}, h_{2}, \ldots, h_{t}\right\}$ denotes the Groebner basis of $I=<f_{1}, f_{2}, \ldots, f_{s}>$ through Buchberger algorithm. Since there is no public Zero point of $f_{1}, f_{2}, \ldots, f_{s} \in K[z]$ in $\square^{2}, h_{t}$ is a non-zero constant via Hilbert Nullstellensatz theorem. According to the Buchberger algorithm, $h_{i}=f_{i}, i=1,2, \ldots s$.

Let $h_{i}, i>s$ is the non-zero residue from the division of some Spoly multiple polynomial with $h_{1}, h_{2}, \ldots, h_{i-1}$ through Buchberger algorithm. Thus, we can get:

$$
h_{i}=\operatorname{Spoly}\left(h_{l_{1}}, h_{l_{2}}\right)-\sum_{j=1}^{i-1} w_{i}^{j} h_{j}
$$

Where, $l_{1} \neq l_{2}$, and $l_{1}, l_{2} \in\{1,2, \ldots, i-1\} . w_{i}^{j}$ is obtained from the division of multiple polynomial. As the definition of Spoly, (8) could be converted into $h_{i}=\sum_{j=1}^{i-1} r_{i}^{j} h_{j}$

Then, we can get:

$$
\begin{aligned}
h_{s+1}= & \sum_{j=1}^{s} r_{i}^{s+1} h_{j} \\
h_{s+2}= & \sum_{j=1}^{s+1} r_{i}^{s+2} h_{j} \\
& \cdots \\
h_{t}= & \sum_{j=1}^{t-1} r_{i}^{t} h_{j}
\end{aligned}
$$

Where, $r_{i}^{j} \in K[z], j=1,2, \ldots t-1, i=s+1, \ldots, t . t$ is input into $h_{t}$ according to the sequence $h_{t-1}, h_{t-2}, \ldots, h_{s+1}$. Then we can get: $h_{t}=\sum_{j=1}^{s} e_{j}^{t} h_{j}=\sum_{j=1}^{s} e_{j}^{t} f_{j} \cdot h_{t}$ is a non-zero constant. And $1=\sum_{j=1}^{s} e_{j}^{t} f_{j} / h_{t}$, then $g_{j}=e_{j}^{t} / h_{t}$. 
$p_{\varepsilon}, \varepsilon \in E$ is a Laurent multiple polynomial. If $p_{\varepsilon}, \varepsilon \in E$ has no zero point in $\left(\square \backslash\{0\}^{2}\right), f_{\varepsilon}=p_{\varepsilon} \square$ denom $\left(p_{\varepsilon}\right), \varepsilon \in E$. Where $\operatorname{denom}\left(p_{\varepsilon}\right)$ presents the denominator of $p_{\varepsilon}$. According to the lemma 3, we can get:

$$
1=\sum_{\varepsilon \in E} f_{\varepsilon} g_{\varepsilon}=\sum_{\varepsilon \in E} p_{\varepsilon} \operatorname{denom}\left(p_{\varepsilon}\right) g_{\varepsilon}
$$

Let $q_{\varepsilon}=0.25 \operatorname{denom}\left(p_{\varepsilon}\right) g_{\varepsilon}, \varepsilon \in E \quad$, then $\sum_{\varepsilon \in E} p_{\varepsilon}\left(z^{2}\right) q_{\varepsilon}\left(z^{2}\right)=0.25$. Thus, $M\left(z_{1}, z_{2}\right)=\sum_{\varepsilon \in E} q_{\varepsilon}\left(z^{-2}\right) z^{\varepsilon}$. However, this $M\left(z_{1}, z_{2}\right)$ cannot meet the $M\left((-1)^{\varepsilon}\right)=\delta_{\varepsilon, 0}$ and the smoothness is inadequate. This paper improve it by:

$$
M\left(z_{1}, z_{2}\right)=\overline{\frac{1}{8^{m}}\left(1+z_{1}\right)^{m}\left(1+z_{2}\right)^{m}\left(1+\frac{1}{z_{1} z_{2}}\right)^{m} H\left(z_{1}, z_{2}\right)}
$$

Where, $m \geq 1 \in \square, H\left(z_{1}, z_{2}\right)$ is a Laurent multiple polynomial.

\subsection{Validation of $\mathrm{H}\left(\mathrm{z}_{1}, \mathrm{z}_{2}\right)$}

As the increasing of $m$, the smoothness of $M\left(z_{1}, z_{2}\right)$ will increase. The validation of $\mathrm{H}\left(\mathrm{z}_{1}, \mathrm{z}_{2}\right)$ should be carried out. Let's define:

$$
M^{\prime}(z)=M(z) \frac{1}{8^{m}}\left(1+z_{1}\right)^{m}\left(1+z_{2}\right)^{m}\left(1+\frac{1}{z_{1} z_{2}}\right)^{m}=\sum_{\varepsilon \in E} p_{\varepsilon}^{\prime}\left(z^{2}\right) z^{\varepsilon}
$$

If $\left\{p_{(0,0)}^{\prime}\left(z_{1}^{2}, z_{2}^{2}\right), p_{(0,1)}^{\prime}\left(z_{1}^{2}, z_{2}^{2}\right), p_{(1,0)}^{\prime}\left(z_{1}^{2}, z_{2}^{2}\right), p_{(1,1)}^{\prime}\left(z_{1}^{2}, z_{2}^{2}\right)\right\}$ has no public zero point in $\left(\square \backslash\{0\}^{2}\right)$, then $\mathrm{H}\left(\mathrm{z}_{1}, \mathrm{z}_{2}\right)$ exits.

Lemma 4. Assume $F(z)$ is the detailed filter operator of compactly support set $f(x)$, which satisfies $f(w)=F(z) f(w / 2)$. Riesz basis could be formed by the movement $\{f(x-k)\}_{k \in \square^{2}}$ of,$f(x)$. Then, $F(z)$ could be expressed as $F(z)=\sum_{\varepsilon \in E} f_{\varepsilon}\left(z^{2}\right) z^{\varepsilon}$. Thus, $\left\{f_{\varepsilon}\left(z^{2}\right), \varepsilon \in E\right\}$ has no public zero point in $\left(\square \backslash\{0\}^{2}\right)$.

Proof. Assume there are public zero point $z^{*}=e^{-i w^{*} / 2}$ in $\left(\square \backslash\{0\}^{2}\right)$, then, $F\left((-1)^{\varepsilon} z^{*}\right)=0, \varepsilon \in E$.

$z^{*}$ is the public zero point of $F\left((-1)^{\varepsilon} z\right)$.

Let $B\left(z^{2}\right)=\sum_{k \in \square^{2}} f(w+2 k \pi) \overline{f(w+2 k \pi)}$

$\therefore$ Riesz basis could be formed by $\{f(x-k)\}_{k \in \square^{2}}$, then, $B\left(z^{2}\right)>0, \forall z \in \square$.

$$
\therefore B\left(z^{2}\right)=\sum_{\varepsilon \in E} F\left((-1)^{\varepsilon} z\right) \overline{F\left((-1)^{\varepsilon} z\right)} B\left((-1)^{\varepsilon} z\right)
$$

$$
\because B\left(z^{*^{2}}\right)=0
$$

That is conflict with the $B\left(z^{2}\right)>0$. So, there is no public zero point of $\left\{f_{\varepsilon}\left(z^{2}\right), \varepsilon \in E\right\}$ in $\left(\square \backslash\{0\}^{2}\right)$. 


\subsection{Duality of the Constructed Wavelet}

The duality of the constructed wavelet ensure that the Riesz basis is in a closed space. If the $M(z)$ is dual: $M\left(z_{1}, z_{2}\right)=M\left(z_{2}, z_{1}\right)$ and $M^{*}(z)$ satisfies:

$$
\sum_{\varepsilon \in E} M\left((-1)^{\varepsilon} z\right) \overline{M^{*}\left((-1)^{\varepsilon} z\right)}=1, M^{*}(z)=\sum_{\varepsilon \in E} q_{\varepsilon}^{*}\left(\frac{1}{z^{2}}\right) z^{\varepsilon} .
$$

Define $q_{\varepsilon}\left(z_{1}^{2}, z_{2}^{2}\right)=\frac{1}{2}\left(q_{\varepsilon}^{*}\left(z_{1}^{2}, z_{2}^{2}\right)+q_{\varepsilon}^{*}\left(z_{2}^{2}, z_{1}^{2}\right)\right)$ and $M^{*}(z)=\sum_{\varepsilon \in E} q_{\varepsilon}\left(\frac{1}{z_{1}^{2}}, \frac{1}{z_{2}^{2}}\right) z^{\varepsilon}$

Where $\bar{\varepsilon}=\left\{\begin{array}{clll}\varepsilon & \varepsilon=(0,0) & \text { or } & (1,1) \\ (1,1)-\varepsilon & \varepsilon=(1,0) & \text { or } & (0,1)\end{array}\right.$

Then, $M(z)$ satisfies: $\sum_{\varepsilon \in E} M\left((-1)^{\varepsilon} z\right) \overline{M\left((-1)^{\varepsilon} z\right)}=1$ and $M\left(z_{1}, z_{2}\right)=M\left(z_{2}, z_{1}\right)$.

Proof.

$$
M\left(z_{1}, z_{2}\right)=\sum_{\varepsilon \in E} q_{\varepsilon}\left(\frac{1}{z_{1}^{2}}, \frac{1}{z_{2}^{2}}\right) z^{\varepsilon}=\sum_{\varepsilon \in E} \frac{1}{2}\left(q_{\varepsilon}^{*}\left(z_{1}^{2}, z_{2}^{2}\right)+q_{\bar{\varepsilon}}^{*}\left(z_{2}^{2}, z_{1}^{2}\right)\right) z^{\varepsilon}=M\left(z_{2}, z_{1}\right)
$$

From $M\left(z_{1}, z_{2}\right)=M\left(z_{2}, z_{1}\right)$, we can get $\sum_{\varepsilon \in E} p_{\varepsilon}\left(z_{1}^{2}, z_{2}^{2}\right) z^{\varepsilon}=\sum_{\varepsilon \in E} p_{\varepsilon}\left(z_{2}^{2}, z_{1}^{2}\right) z^{\bar{\varepsilon}}$.

Due to the uniqueness of $M\left(z_{1}, z_{2}\right)$, we can get:

$$
\begin{aligned}
& p_{\varepsilon}\left(z_{1}^{2}, z_{2}^{2}\right)=p_{\varepsilon}\left(z_{1}^{2}, z_{2}^{2}\right) \\
& \text { Then, } \sum_{\varepsilon \in E} M\left((-1)^{\varepsilon} z\right) \overline{M^{*}\left((-1)^{\varepsilon} z\right)}=1 \Leftrightarrow \sum_{\varepsilon \in E} p_{\varepsilon}\left(z_{1}^{2}, z_{2}^{2}\right) q_{\varepsilon}^{*}\left(z_{1}^{2}, z_{2}^{2}\right)=0.25 \\
& \sum_{\varepsilon \in E} M\left((-1)^{\varepsilon} z\right) \overline{M\left((-1)^{\varepsilon} z\right)}=4 \sum_{\varepsilon \in E} p_{\varepsilon}\left(z_{1}^{2}, z_{2}^{2}\right) q_{\varepsilon}^{*}\left(z_{1}^{2}, z_{2}^{2}\right) \\
& =2 \sum_{\varepsilon \in E} p_{\varepsilon}\left(z_{1}^{2}, z_{2}^{2}\right)\left(\sum_{\varepsilon \in E} q_{\varepsilon}^{*}\left(z_{1}^{2}, z_{2}^{2}\right)+q_{\varepsilon}^{*}\left(z_{2}^{2}, z_{1}^{2}\right)\right)=1
\end{aligned}
$$

From the above proof, it is observed that the constructed wavelet is dual

\section{Applications of the Constructed Dual Wavelet in Image Edge Detection}

The application of the constructed dual wavelet is presented by using image edge detection for example to demonstrate how the constructed dual wavelet is able to outperform the detection comparing with Sobel and Canny approach [12]. The edge of an image is a basic characteristics which is very important for image processing. The image division is heavily based on the edge detection.

Due to the gray degree of an image in the edge has great change. The wavelet is compactly support set which may have multi-scale analysis ability. Let $V_{j}=\overline{\operatorname{span}\left\{\phi\left(2^{j} x-k\right), k \in \sqcup^{2}\right\}}, j \in \sqcup$ forms multi-scale analysis in $\left\{V_{j}\right\}_{j \in \square}$.

Assume the scale space $V_{j+1}$ has the dual decomposition : $V_{j+1}=V_{j} \oplus W_{j}$, where $W_{j}$ is the orthogonal complement space. Using the dual wavelet proposed in this paper, we carry out some experiments. The fingerprints with two types feature are used for this experimental applications. The proposed dual wavelet manner is compared with Sobel and Canny for detecting the edge of the fingerprint images. 
Let an image expressed as $\{f(m, n) \mid m=0, \ldots, M, n=0, \ldots, N\}$, the wavelet transfer with the scale of $2^{j}$ and it is marked as $W_{2^{j}}^{\varepsilon} f(m, n), \varepsilon \in E$. For making full use of the information obtained from the dual wavelet transfer in certain scale, the transfer mod could be given:

$$
\begin{aligned}
& a_{2^{j}}(m, n)=W_{2^{j}}^{(0,1)} f(m, n)+W_{2^{j}}^{(1,1)} f(m, n) \\
& b_{2^{j}}(m, n)=W_{2^{j}}^{(1,0)} f(m, n)+W_{2^{j}}^{(1,1)} f(m, n)
\end{aligned}
$$

The dual wavelet transfer mode of pixel $(m, n)$ could be:

$M_{2^{j}} f(m, n)=\sqrt{\left|a_{2^{j}}(m, n)\right|^{2}+\left|b_{2^{j}}(m, n)\right|^{2}}$, the corresponding argument is:

$$
A_{2^{j}} f(m, n)=\arg \left(a_{2^{j}}(m, n)+i b_{2^{j}}(m, n)\right) \text {. }
$$

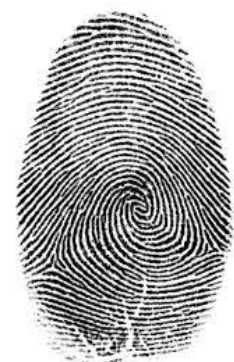

(a) Original

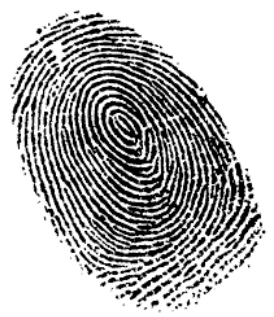

(e) Original

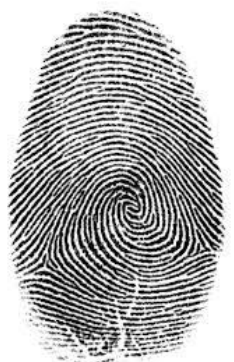

(b) Sobel

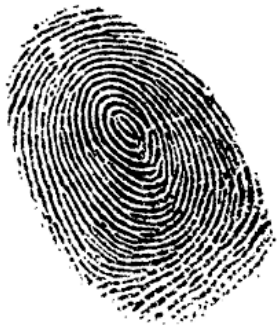

(f) Sobel

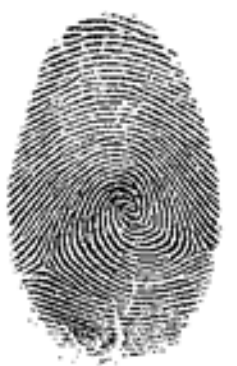

(c) Canny

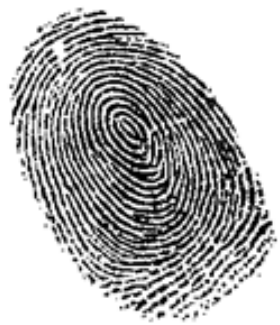

(g) Canny

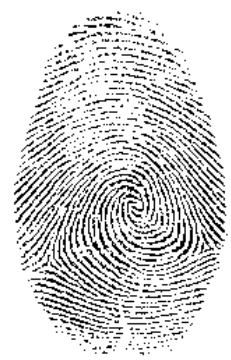

(d) Dual Wavelet

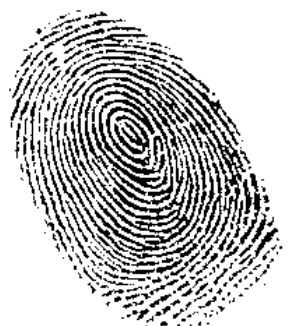

(h) Dual

Wavelet

Figure 1. Application Results

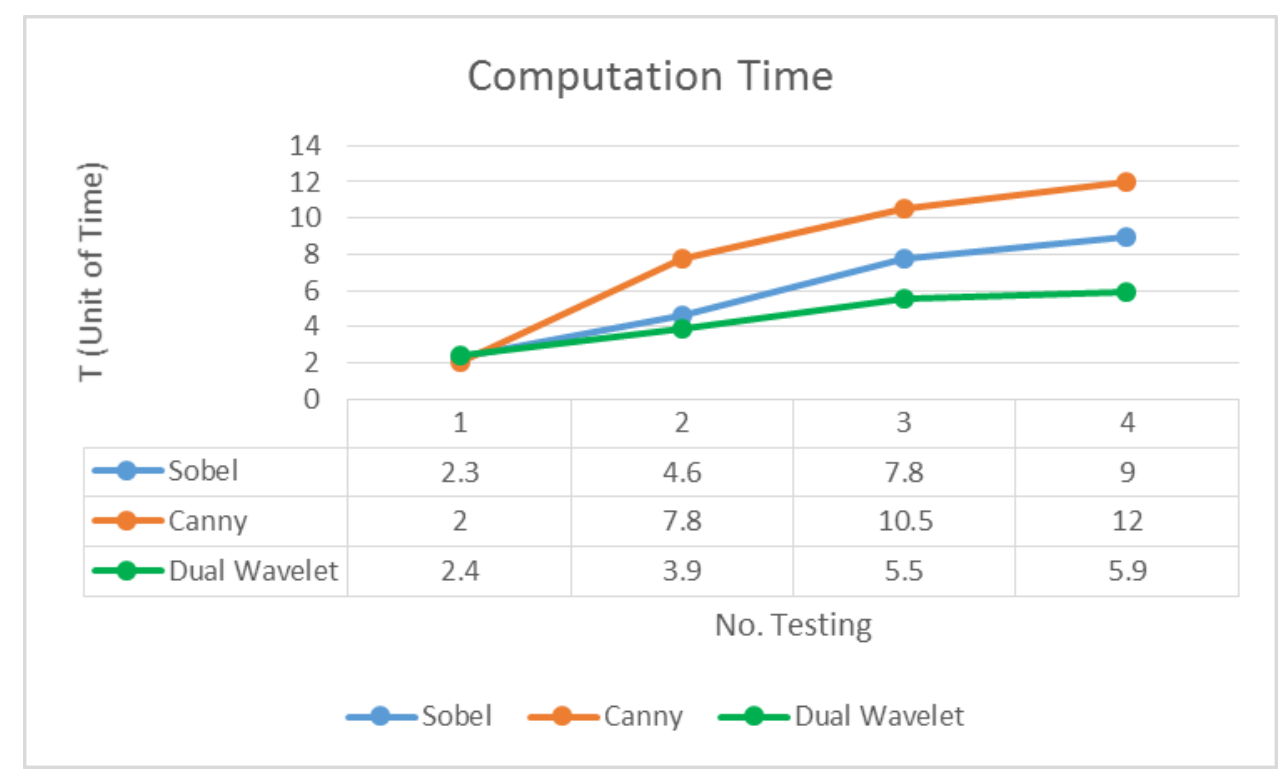

Figure 2. Computation Results 
Each pixel is with 8 near pixel, then, the argument has eight directions. Along with the direction obtained from argument normalization, the maximum value of the mod from wavelet transfer. The maximum value is able to get the image edge. The following figure 1 shows the results from the experiments. Figure 1 (a) and (e) shows two types of fingerprint images for using different edge detection approach to get the best processing results. Figure 1 (b) and (f) are the results from using Sobel filter. It could be observed that the images are most of the same as the original images since the Sobel filter uses $3 \times 3$ kernels which are convolved with the original image to calculate approximations of the derivatives: one for horizontal changes, and one for vertical.

Figure 1. (c) and (g) are from the results of using Canny edge detector which uses a multi-stage algorithm to detect a wide range of edges in images. From the application experiments in fingerprint, it is observed that this approach performs not well as the Sobel filter. The results look unclear due to the number of parameters which takes much more computation time. And the effectiveness of this method is lagged too. Figure 1 (d) and (h) are the results using the proposed dual wavelet approach, which uses 8 directions to calculate the edge pixel. The results -Figure 1 and Figure 2 from this application outperforms Sobel and Canny methods in terms of computation and edge detection effectiveness.

\section{Conclusions}

This paper introduce a construction of a dual wavelet through the compactly support set/functions which can form the Riesz basis. Based on the mathematic presentation of the construction of the dual wavelet, which is a dual orthogonal with scale function and duality, the constructed dual wavelet is used for an application testing with fingerprint. The test is for edge detection. The proposed approach is compared with Sobel and Canny which are widely used in edge detection. It is observed that from the results, the proposed dual wavelet outperforms the Sobel and Canny filter in terms of effectiveness and computation time.

Future research directions could be carried out in two aspects. Firstly, the constructed dual wavelet is required to test its freedom degree so that the compactly support function is able to form the high dimensional wavelet basis (HDWB). With the HDWB, the constructed dual wavelet could be used for high dimensional data processing such as Big Data analytics. Secondly, more applications will be carried out for examining the feasibility and practicality of the proposed dual wavelet not only in image processing with other high dimensional pixels, but also in other domains like 3-D object tracking and tracing.

\section{References}

[1] S. Mallat, "A wavelet tour of signal processing: Academic press", (1999).

[2] S. G. Mallat, "A theory for multiresolution signal decomposition: the wavelet representation", IEEE Transactions on Pattern Analysis and Machine Intelligence, vol. 11, (1989), pp. 674-693.

[3] D. E. Newland, "An introduction to random vibrations, spectral \& wavelet analysis: Courier Dover Publications, (2012).

[4] I. I. A. Novikov, V. I. U. e. Protasov, and M. A. Skopina, "Wavelet theory, 239: American Mathematical Soc., (2011).

[5] G. Bhutada, R. Anand, and S. Saxena, "Edge preserved image enhancement using adaptive fusion of images denoised by wavelet and curvelet transform", Digital Signal Processing, vol. 21, (2011), pp. 118130.

[6] G. Anbarjafari and H. Demirel, "Image super resolution based on interpolation of wavelet domain high frequency subbands and the spatial domain input image", ETRI journal, vol. 32, (2010), pp. 390-394.

[7] X. You, L. Du, Y.-m. Cheung, and Q. Chen, "A blind watermarking scheme using new nontensor product wavelet filter banks", IEEE Transactions on Image Processing, vol. 19, (2010), pp. 3271-3284. 
[8] R. Y. Zhong, G. Q. Huang, Q. Y. Dai, and T. Zhang, "Mining SOTs and Dispatching Rules from RFIDenabled Real-time Shopfloor Production Data", Journal of Intelligent Manufacturing, vol. 25, (2014), pp. 825-843.

[9] R. Y. Zhong, G. Q. Huang, and Q. Y. Dai, "A Big Data Cleansing Approach for n-dimensional RFIDCuboids", Proceeding of the 2014 IEEE 18th International Conference on Computer Supported Cooperative Work in Design (CSCWD), (2014) 21-23 May, Taiwan.

[10] R. Y. Zhong, Z. Li, A. L. Y. Pang, Y. Pan, T. Qu, and G. Q. Huang, RFID-enabled Real-time Advanced Planning and Scheduling Shell for Production Decision-making, International Journal of Computer Integrated Manufacturing, vol. 26, (2013), pp. 649-662.

[11] R. Y. Zhong, Q. Y. Dai, T. Qu, G. J. Hu, and G. Q. Huang, "RFID-enabled Real-time Manufacturing Execution System for Mass-customization Production", Robotics and Computer-Integrated Manufacturing, vol. 29, (2013), pp. 283-292.

[12] E. Schiavi, J. F. Garamendi, and A. Martín, "Medical Image Processing: Mathematical Modelling and Numerical Resolution", in Advances in Numerical Simulation in Physics and Engineering, ed: Springer, (2014).

\section{Author}

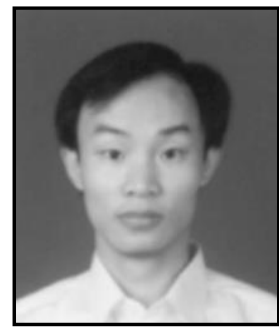

Yubin Li, he is a lecturer in Gannan Normal University. He graduated from Mathematics and Computer Science \& Technology, Gannan Normal University with bachelor degree in 2004 and master degree in software engineering from Yunnan University in 2009 respectively. His research direction includes image processing, computer applications, information management. He has published several papers in international/national journals and conference. 